\title{
Studies on Direct and Indirect Effects of Different Plant Characters on Yield of Taro (Colocasia esculenta L. Schott) Var. Antiquorum
}

\author{
K. K. Paul* and M. A. Bari \\ Institute of Biological Sciences, Rajshahi University, Rajshahi-6205, Bangladesh \\ *Corresponding author and Email: krshnnd@yahoo.com,mabarimiahbd@yahoo.com
}

Received: 23 April $2011 \quad$ Accepted: 26 November 2011

\begin{abstract}
Thirty accessions of taro (Colocasia esculenta L) varieties were evaluated to estimate the direct and indirect effects of different plant characters, namely, plant height, petiole length, petiole breadth, leaf number, leaf length, leaf breadth, LAI, inflorescence length, peduncle length, corm length, corm breadth, cormel number, cormel length, cormel breadth, corm weight, cormel weight on yield per plant. Plant height, petiole length, corm breadth, cormel number, cormel length exhibited direct effect on yield per plant at the genotypic level. Cormel weight and cormel breadth had the highest direct effect on yield per plant at the phenotypic level. The residual effect was 0.3043 at the genotypic level and that at phenotypic level was 0.4874 .
\end{abstract}

\section{Keywords: Colocasia esculenta, direct effect, indirect effect, yield of taro}

\section{Introduction}

Taro (Colocasia esculenta L. Schott), locally called mukhikachu belongs to the family Araceae. Cultivated taro is classified as Colocasia esculenta but the species is considered to be polymorphic. There are at least two botanical varieties (Purseglove, 1972) of taro, of which Colocasia esculenta (L.) Schott var. antiquorum (Schott) is synonymous with $C$. esculenta var. globulifera Engl. \& Krause. It is used as food (for animal and human beings), medicine, ornament and industrial raw material. The whole part of this plant (corm, cormels, stalk, inflorescence and flowers) can be used as food. Coursey (1968) gave the composition of corms as water (63-85 percent), protein (1.3-4.0 percent), fat (2.0-4.0 percent), carbohydrate (13 29 percent), fibre (0.6-1.2 percent), ash (0.6-1.3 percent) and vitamin B \& C (in appreciable quantities). It is also a rich source of calcium, phosphorus, iron, thiamine, riboflavin and niacin, which are important constituents of human diet.
The world's rapid population growth is demanding increased production and greater diversification of crops. Roots and tubers can play a major role in addressing this issue, including edible aroids which in many countries are treated just as a vegetable. With the advent of modern agriculture, introduction of high yielding crop varieties, the underutilized crops like aroids are pushed under the threat of extinction. Some varieties of edible aroids are endemic in nature, being cultivated in particular areas of the country, their merits were not evaluated and their proper taxonomic positions were not properly determined. In spite of tremendous potentialities, the existing edible aroids are running in vulnerable condition and without being properly evaluated. On this perspective, this present piece of research work was undertaken to make a scientific evaluation of the mukhikachu cultivars growing in the country covering the aspects of biometrical characterization. Specifically, the present investigation was designed to 
estimate the direct and indirect effects of different plant characters for identification of genotypes from cultivars of Colocasia esculenta var antiquorum (mukhikachu) for tuber yield.

\section{Materials and Methods}

\subsection{Plant materials}

Four hundred and fifty seven accessions of edible mukhikachu were collected from aroid growing thirteen districts of Bangladesh in 20052006. They were maintained at the experimental farm of the Institute of Biological sciences in 2005-2006 at Rajshahi University, Bangladesh. The accessions were grown in loamy soil in a single row of 4 meters length with inter row spacing of $60 \mathrm{~cm}$. The present biometrical investigation was conducted with collected 30 elite accessions of mukhikachu from different aroid growing pockets in the country, such as Arani, Godagari and Meher chandi of Rajshahi, Tala of Satkhira, Churamonkati and of chougacha of Jessore, Santhahar of Bogra, Panchbibi of Joypurhat, Munshiganj and Joydebpur of Dhaka, Madhupur of Mymensingh and sadar upozilla of Barisal in Bangladesh.

\subsection{Site, soils and seasons}

This investigation was conducted at the experimental farm of the Institute of Biological Sciences research field at Rajshahi University, Rajshahi during the on set of rainy season 20052006. The land in which the experiment was carried out was medium high. The soil was part of Level Barind agroecological zone marked by sandy loam with $\mathrm{p}^{\mathrm{H}} 6.5$. The rainfall distribution in rabi season was very low or Scanty $(<40 \mathrm{~cm})$. So that at least three times flood irrigation were required. All recommended agricultural practices were followed during production period. The propagules were sown on March, 2006. Two healthy propagules were planted per hill during plantation and finally, a single healthy plant was retained.

\subsection{Experimental design}

The experiment was set up in a Randomized Complete Block (RCB) design with three replications. In each experimental plot plant propagules of mukhikachu were planted with row to row spacing of $0.75 \mathrm{~m}$ and plant to plant spacing of $0.60 \mathrm{~cm}$.

\subsection{Data recording and harvesting}

When the vegetative growth was in climax or was slowed down, leaves become yellowish, dry and dropping then the quantitative parameters were observed and data were recorded following descriptors of taro with necessary modifications (IPGRI, 1999). Data on morphological characters were recorded from randomly selected five plants from each accession between 170 days after planting, while yield and other characters were recorded at harvest. Leaf observations were made on two fully developed leaves per plant and the average of three plants was recorded. Measurements were as follows:

Plant height (PLH): Plant height $(\mathrm{cm})$ was measured from soil surface to tip of the terminal leaf.

Petiole length (PEL): The length between the base of the plant and point of insertion or junction of leaf expressed (in $\mathrm{cm}$ ).

Petiole breadth (PEB): Average measurements were taken for three position of petiole such as petiole base, middle position and junction of leaf attachment (in $\mathrm{cm}$ ).

Leaf length (LEL): The leaf length was measured from leaf base to leaf apex (in $\mathrm{cm}$ ).

Leaf breadth ( $L E B)$ : The cross length of one end to the other of leaf lamina (in $\mathrm{cm}$ ).

Leaf Number (LEN): Total number of leaves per plant was counted from bottom to the top of the randomly selected five plants.

Leaf area Index (LAI): The leaf area index was measured as leaf length $\mathrm{x}$ leaf breadth $\mathrm{x} 0.75$ (Montgomery, 1911). 
Inflorescence length (IFL): It was measured as the total length of inflorescence from peduncle base to the tip of the inflorescence (in $\mathrm{cm}$ ).

Peduncle length (PDL): The stem holding the whole inflorescence is called peduncle and was measured as the length between peduncle base and starting portion of spathe (in $\mathrm{cm}$ ).

Corm length $(C R L)$ : The length of corm was measured with a scale or measuring tape through vertically (in $\mathrm{cm}$ ).

Corm breadth $(C R B)$ : Breadth of the rhizome was measured horizontally through the middle position of corm (in $\mathrm{cm}$ ).

Cormel length $(C O L)$ : The length of cormel was measured with scale or tape through vertically (in $\mathrm{cm}$ ).

Cormel breadth (COB): Breadth of the cormel was measured horizontally through the middle position of corm (in $\mathrm{cm}$ ).

Cormel number (CON): The number of cormels present or attached adjacent to the corm after harvesting.

Cormel weight $(\mathrm{COW})$ : After detaching the cormlet from corm weight was measured (in gm).

Corm weight $(C R W)$ : After harvesting the corm was weighted (in gm).

Yield per plant (YPP): It was estimated as the total weight of corm and cormels (in gm).

\subsection{Statistical analysis}

The collected data were analyzed following the biometrical techniques of analysis developed by Mather (1949) based on mathematical model of Fisher et al. (1932) using the SPSS and Excel software. Mean and Critical differences were worked out by the method of analysis of variance. The analysis of variance for each character under the study was performed by $\mathrm{F}$ Test (Cochran and Cox, 1960). Critical differences were calculated to compare between any two means following (Panse and Suhatme, 1978). For path coefficient analysis, the analysis of both variance and covariance viz. genotypic variance $\left(\sigma_{g}^{2}\right)$, phenotypic variance $\left(\sigma_{p}^{2}\right)$, and environment variance $\left(\sigma_{\mathrm{e}}^{2}\right)$ were calculated between all possible pairs of characters separately using the formula developed by Singh and Chaudhary (1977). The path coefficient (direct and indirect efects) analysis was done following Wright $(1921,1923)$ formula extended by Dewey and Lu (1959).

\section{Results and Discussion}

The results on direct and indirect effects of yield contributing characters on yield per plant both at genotypic and phenotypic levels are presented in Table 1 and Table 2, respectively.

\subsection{At genotypic level}

At genotypic level, plant height had highly positive and direct effect on yield per plant (1.2258). Positive and indirect effects also found via petiole breadth (0.1370), leaf number (0.1424), inflorescence length (0.1755), corm length (0.1026), corm breadth (0.1736), cormel number (0.1327) and cormel length (0.3910). Petiole length showed low and positive direct effect on yield per plant (0.3167) at the genotypic level. Indirect but positive effects were observed through plant height $(0.2206)$, petiole breadth (0.1644), leaf number (0.1609), leaf area index (0.1482), inflorescence length (0.1946), peduncle length (0.1194), corm length (0.1062), corm breadth $(0.1751)$, cormel number $(0.1343)$, cormel weight (0.1122) and cormel length (0.4097). High and positive direct effect of petiole breadth was observed on yield per plant $(0.7610)$ at genotypic level. Low and indirect effects of plant height (0.2206), leaf number (0.1672), inflorescence length (0.2038), corm length (0.1114), corm breadth (0.1836), cormel number $(0.1403)$ and cormel length (0.4333) were also found on yield. At the genotypic level, leaf length exhibited low and positive direct effects on yield per plant (0.3064). Indirect and positive effects were also found via plant height $(0.3015)$, petiole breadth (0.2230), leaf number (0.2117), leaf area index (0.0940), corm length (0.1406), corm breadth (0.2266), cormel number (0.3264), cormel weight (0.0645) and cormel length (0.5202). Leaf number exhibited low positive and direct effect $(0.3179)$ on yield per plant. 
Table 1. Direct (diagonal bold) and indirect effects of different plant characters on yield per plant of three cultivars of mukhikachu (Colocasia esculenta L.) at genotypic level

\begin{tabular}{|c|c|c|c|c|c|c|c|c|c|c|c|c|c|c|c|c|c|}
\hline $\begin{array}{c}\text { Character } \\
\text { s }\end{array}$ & PLH & PEL & PEB & LEL & LEB & LEN & LAI & IFL & PDL & CRL & CRB & CRW & $\mathrm{CON}$ & COW & COL & $\mathrm{COB}$ & $\mathrm{R}_{\mathrm{g}}$ YPP \\
\hline PLH & 1.2258 & 0.0570 & 0.1370 & 0.0754 & 0.0033 & 0.1424 & 0.0987 & 0.1755 & 0.0717 & 0.1026 & 0.1736 & 0.0238 & 0.1327 & 0.0485 & 0.3910 & 0.0354 & -0.349 \\
\hline PEL & 0.2206 & 0.3167 & 0.1644 & 0.0870 & 0.0039 & 0.1609 & 0.1482 & 0.1946 & 0.1194 & 0.1062 & 0.1751 & 0.0244 & 0.1343 & 0.1122 & 0.4097 & 0.0363 & 0.387 \\
\hline PEB & 0.2206 & 0.0684 & 0.7610 & 0.0898 & 0.0039 & 0.1672 & 0.0806 & 0.2038 & 0.0710 & 0.1114 & 0.1836 & 0.0230 & 0.1403 & 0.0501 & 0.4333 & 0.0387 & -0.407 \\
\hline LEL & 0.3015 & 0.0899 & 0.2230 & 0.3064 & 0.0052 & 0.2117 & 0.0940 & 0.0100 & 0.0717 & 0.1406 & 0.2266 & 0.0326 & 0.3264 & 0.0645 & 0.5202 & 0.0475 & $0.920 * *$ \\
\hline LEB & 0.2268 & 0.0694 & 0.1674 & 0.0907 & 0.0176 & 0.1669 & 0.0719 & 0.0439 & 0.1282 & 0.1072 & 0.1928 & 0.0269 & 0.1494 & 0.1277 & 0.4370 & 0.0397 & 0.409 \\
\hline LEN & 0.5491 & 0.1602 & 0.4003 & 0.2041 & 0.0092 & 0.3179 & 0.1290 & 0.2056 & 0.0691 & 0.2105 & 0.3226 & 0.0500 & 0.2476 & 0.0917 & 0.7709 & 0.0705 & $0.840^{* *}$ \\
\hline LAI & 0.8409 & 0.3262 & 0.4261 & 0.2001 & 0.0088 & 0.2144 & 0.1439 & 0.1962 & 0.0578 & 0.0810 & 0.4033 & 0.0291 & 0.2973 & 0.2772 & 0.5586 & 0.0845 & $0.650^{* * *}$ \\
\hline IFL & 1.0297 & 0.2948 & 0.7419 & 0.0643 & 0.0173 & 0.2985 & 0.0626 & 0.2090 & 0.1408 & 0.2503 & 0.5139 & 0.0289 & 0.4057 & 0.1426 & 1.2303 & 0.0949 & $0.640 * *$ \\
\hline PEL & 0.6987 & 0.3006 & 0.4299 & 0.1747 & 0.0179 & 0.1748 & 0.0662 & 0.2341 & 0.1257 & 0.1332 & 0.3380 & 0.0572 & 0.5718 & 0.1732 & 0.8193 & 0.0601 & $0.810^{* *}$ \\
\hline CRL & 0.5124 & 0.1371 & 0.3455 & 0.1756 & 0.0077 & 0.2727 & 0.0475 & 0.2503 & 0.0683 & 0.2454 & 0.1744 & 0.0286 & 0.1198 & 0.0352 & 0.4271 & 0.0394 & $0.714 * *$ \\
\hline CRB & 0.2770 & 0.0722 & 0.1819 & 0.0904 & 0.0044 & 0.1335 & 0.0755 & 0.1398 & 0.0553 & 0.0461 & 0.7682 & 0.0113 & 0.0313 & 0.0293 & 0.5040 & 0.0145 & 0.357 \\
\hline CRW & 0.5406 & 0.1435 & 0.3249 & 0.1854 & 0.0088 & 0.2947 & 0.0777 & 0.1120 & 0.1333 & 0.1303 & 0.1613 & 0.0540 & 0.1322 & 0.0394 & 0.4742 & 0.0430 & $0.777 * *$ \\
\hline $\mathrm{CON}$ & 0.3015 & 0.0789 & 0.1978 & 0.1008 & 0.0049 & 0.1459 & 0.0793 & 0.1572 & 0.1157 & 0.0545 & 0.0446 & 0.0132 & 0.5395 & 0.0080 & 0.3203 & 0.0178 & 0.401 \\
\hline COW & 0.2231 & 0.1333 & 0.1431 & 0.0741 & 0.0084 & 0.1094 & 0.1496 & 0.1118 & 0.0817 & 0.0324 & 0.0845 & 0.0080 & 0.0162 & 0.2665 & 0.1303 & 0.0112 & $0.756^{* *}$ \\
\hline $\mathrm{COL}$ & 0.3861 & 0.1045 & 0.2656 & 0.1284 & 0.0062 & 0.1974 & 0.0648 & 0.2071 & 0.0830 & 0.2643 & 0.3119 & 0.0206 & 0.1392 & 0.0280 & 1.2414 & 0.0258 & $0.481^{*}$ \\
\hline $\mathrm{COB}$ & 0.4621 & 0.1222 & 0.3135 & 0.1547 & 0.0074 & 0.2384 & 0.1295 & 02111 & 0.0805 & 0.1028 & 0.1183 & 0.0247 & 0.1020 & 0.0317 & 0.3414 & 0.0940 & $0.609 * *$ \\
\hline
\end{tabular}

Residual effects $=0.3043, *$ Significant at 0.05 level $* *$ significant at 0.01 level

$\mathrm{PLH}=$ Plant height, PEL = Petiole length, PEB= Petiole breadth, LEL= Leaf length, LEB= Leaf breadth, LEN= Leaf Number, LAI= Leaf area Index, IFL= Inflorescence length, $\mathrm{PDL}=$ Peduncle length, $\mathrm{CRL}=\mathrm{Corm}$ length, $\mathrm{CRB}=\mathrm{Corm}$ breadth, $\mathrm{COL}=\mathrm{Cormel}$ length, $\mathrm{COB}=\mathrm{Cormel}$ breadth, $\mathrm{CON}=\mathrm{Cormel}$ number, $\mathrm{CRW}=\mathrm{Corm}$ weight, $\mathrm{YPP}=$ Yield per plant 
Table 2. Direct (diagonal) and indirect effects of different plant characters on yield per plant of three cultivars of mukhikachu (Colocasia esculenta L.Schott.) at the phenotypic level

\begin{tabular}{|c|c|c|c|c|c|c|c|c|c|c|c|c|c|c|c|c|c|}
\hline $\begin{array}{l}\text { Character } \\
\mathrm{s}\end{array}$ & PLH & PEL & PEB & LEL & LEB & LEN & LAI & IFL & PDL & CRL & CRB & CRW & $\mathrm{CON}$ & COW & $\mathrm{COL}$ & $\mathrm{COB}$ & $\begin{array}{l}\mathrm{Rp} \\
\mathrm{YPP}\end{array}$ \\
\hline PLH & 0.2823 & 0.1252 & 0.1924 & 0.0253 & 0.2328 & 0.4058 & 0.0271 & 0.2009 & 0.1040 & 0.1221 & 0.0863 & 0.0293 & 0.0714 & 0.1520 & 0.0740 & 0.4102 & 0.349 \\
\hline PEL & 0.1522 & 0.2322 & 0.2033 & 0.0270 & 0.2481 & 0.4375 & 0.0235 & 0.3030 & 0.0408 & 0.1351 & 0.0997 & 0.0320 & 0.0802 & 0.3937 & 0.0843 & 0.4611 & 0.387 \\
\hline PEB & 0.1598 & 0.1389 & 0.3400 & 0.0298 & 0.2542 & 0.4064 & 0.0244 & 0.2621 & 0.0563 & 0.1388 & 0.1028 & 0.0334 & 0.0835 & 0.1809 & 0.0841 & 0.4705 & 0.407 \\
\hline LEL & 0.1971 & 0.1728 & 0.2794 & 0.0362 & 0.3215 & 0.1726 & 0.0246 & 0.3472 & 0.0796 & 0.1812 & 0.1333 & 0.0430 & 0.2510 & 0.2273 & 0.1114 & 0.6153 & $0.750^{* * *}$ \\
\hline LEB & 0.1629 & 0.1428 & 0.2142 & 0.0289 & 0.4034 & 0.4255 & 0.0129 & 0.2880 & 0.0660 & 0.1394 & 0.1021 & 0.0340 & 0.0845 & 04150 & 0.0869 & 0.4815 & 0.409 \\
\hline LEN & 0.2854 & 0.2531 & 0.3835 & 0.0156 & 0.4276 & 0.4014 & 0.0553 & 0.1668 & 0.0854 & 0.0903 & 0.2206 & 0.0250 & 0.1768 & 0.3770 & 0.1801 & 0.9762 & $0.840^{* * *}$ \\
\hline LAI & 0.3388 & 0.2415 & 0.3671 & 0.0373 & 0.2300 & 0.1954 & 0.0226 & 0.4017 & 0.0505 & 0.1080 & 0.2158 & 0.0361 & 0.1904 & 0.7829 & 0.0733 & 0.8220 & $0.859^{* * *}$ \\
\hline IFL & 0.1666 & 0.2067 & 0.2618 & 0.0370 & 0.3413 & 0.1967 & 0.0267 & 0.3404 & 0.0544 & 0.0805 & 0.3769 & 0.0263 & 0.2716 & 0.5017 & 0.1294 & 0.8533 & $0.820^{* *}$ \\
\hline PEL & 0.3024 & 0.0975 & 0.1836 & 0.0297 & 0.2743 & 0.3532 & 0.0118 & 0.1906 & 0.0971 & 0.1060 & 0.3539 & 0.0440 & 0.2573 & 0.7829 & 0.1277 & 0.5715 & $0.818^{* * *}$ \\
\hline CRL & 0.1756 & 0.1598 & 0.2403 & 0.0334 & 0.2864 & 0.1846 & 0.0124 & 0.0805 & 0.0524 & 0.1963 & 0.1960 & 0.0534 & 0.1567 & 0.3512 & 0.1417 & 0.7852 & $0.714^{* *}$ \\
\hline CRB & 0.0711 & 0.0676 & 0.1020 & 0.0141 & 0.1202 & 0.2585 & 0.0142 & 0.3745 & 0.1003 & 0.1123 & 0.3426 & 0.0260 & 0.0810 & 0.2432 & 0.0575 & 0.3883 & 0.357 \\
\hline CRW & 0.1920 & 0.1725 & 0.2641 & 0.0362 & 0.3187 & 0.2328 & 0.0189 & 0.2077 & 0.0993 & 0.2450 & 0.2069 & 0.0430 & 0.1640 & 0.3770 & 0.1497 & 0.8376 & $0.777^{* * *}$ \\
\hline $\mathrm{CON}$ & 0.0802 & 0.0741 & 0.1129 & 0.0158 & 0.1356 & 0.2822 & 0.0173 & 0.3677 & 0.1155 & 0.1223 & 0.1103 & 0.0281 & 0.2515 & 0.2143 & 0.0763 & 0.4149 & 0.401 \\
\hline COW & 0.0565 & 0.1203 & 0.0809 & 0.0108 & 0.2203 & 0.1991 & 0.0233 & 0.2247 & 0.1039 & 0.0907 & 0.1117 & 0.0213 & 0.0709 & 0.7601 & 0.0530 & 0.3045 & $\begin{array}{c}0.756 \\
* *\end{array}$ \\
\hline $\mathrm{COL}$ & 0.1194 & 0.1119 & 0.1635 & 0.0231 & 0.2005 & 0.4094 & 0.0095 & 0.2519 & 0.0709 & 0.2775 & 0.1127 & 0.0368 & 0.1097 & 0.2303 & 0.1749 & 0.5730 & $0.481 *$ \\
\hline $\mathrm{COB}$ & 0.1479 & 0.1368 & 0.2043 & 0.0285 & 0.2481 & 0.5006 & 0.0237 & 0.3813 & 0.0709 & 0.1969 & 0.1699 & 0.0461 & 0.1333 & 0.2957 & 0.1280 & 0.7828 & $0.609^{* * *}$ \\
\hline
\end{tabular}

Residual effect $=0.487$ * Significant at 0.05 level $* *$ significant at 0.01 level, $\mathrm{PLH}=$ Plant height, $\mathrm{PEL}=$ Petiole length, PEB= Petiole breadth, $\mathrm{LEL}=\mathrm{Leaf}$ length, $\mathrm{LEB}=$ Leaf breadth, LEN $=$ Leaf Number, LAI $=$ Leaf area Index, IFL $=$ Inflorescence length, $P D L=$ Peduncle length, $C R L=C$ orm length, $C R B=C$ Corm breadth, $C O L=C o r m e$ length, $\mathrm{COB}=$ Cormel breadth, $\mathrm{CON}=\mathrm{Cormel}$ number, $\mathrm{CRW}=\mathrm{Corm}$ weight, $\mathrm{YPP}=$ Yield per plant 
It showed indirect and positive effects via plant height $(0.5491)$, petiole length $(0.1602)$, petiole breadth (0.4003), leaf length $(0.2041)$, leaf area index (0.1290), inflorescence length (0.2056), corm length (0.2105), corm breadth (0.3226), cormel number (0.2476), cormel length (0.7709) and in genotypic level. Leaf area index showed low and positive direct effect $(0.1439)$ on yield per plant at genotypic level. It also showed positive indirect effect through plant height (0.8409), petiole length $(0.3262)$, petiole breadth (0.4261), leaf length $(0.2001)$, leaf number (0.2144), inflorescence length (0.1962), corm breadth (0.4033), cormel number (0.2973), cormel weight $(0.2772)$ and cormel length $(0.5586)$. Low and positive direct effects of inflorescence length (0.2090) on yield per plant were observed at the genotypic level. Inflorescence length showed positive and indirect effects via plant height (1.0297), petiole length (0.2948), petiole breadth (0.7419), leaf length $(0.0643)$, leaf breadth (0.0173), leaf number $(0.2985)$, leaf area index (0.0626), peduncle length (0.1408), corm length (0.2503), corm breadth (0.5139), cormel number (0.4057), cormel weight (0.1426) and cormel length (1.2303). At the genotypic level peduncle length showed low and positive direct effect (0.1257) on yield per plant. It also showed positive and indirect effect via plant height (0.6987), petiole length (0.3006), petiole breadth (0.4299), leaf length $(0.1747)$, leaf number (0.1748), corm length $(0.1312)$, corm breadth (0.3380), cormel number (0.5718), cormel weight (0.1732) and cormel length (0.8193). Corm length showed low and positive direct effect (0.2454) in genotypic level. It also showed positive and indirect effects via plant height (0.5124), petiole length $(0.1371)$, petiole breadth (0.3455), leaf length $(0.1756)$, leaf number (0.2727), inflorescence length (0.2503), corm breadth (0.1744), cormel number (0.1198) and cormel length $(0.4271$ Corm breadth exhibited high and positive direct effect $(0.7682)$ on yield per plant in genotypic level. In genotypic level, corm weight showed very low and positive direct effect (0.0540) on yield per plant. Positive and indirect effects were found via plant height (0.5406), petiole length $(0.1435)$, petiole breadth (0.3249), leaf length (0.1854), leaf number (0.2947), inflorescence length (0.1120), peduncle length (0.1333), corm length (0.1303), corm breadth (0.1633), cormel number $(0.1322)$ and cormel length (0.4742). Cormel number exhibited highly positive and direct effect $(0.5395)$ on yield per plant. It showed indirect effects via plant height $(0.3015)$, petiole breadth $(0.1978)$, leaf length (0.1008), leaf number (0.1459), inflorescence length (0.1572), peduncle length (0.1157) and cormel length (0.3203) in genotypic level. Cormel number showed low and positive direct effect (0.2515) on yield per plant in phenotypic level. It showed also positive and indirect effects via petiole breadth (0.1129), leaf breadth (0.1356), leaf number (0.2822), inflorescence length (0.3677), peduncle length (0.1155), corm length (0.1223), corm breadth (0.1103), cormel weight $(0.2143)$ and cormel breadth (0.4149).Cormel weight exhibited low and positive direct effects $(0.2665)$ on yield per plant. It showed also positive and indirect effects at the genotypic level on yield per plant via plant height $(0.2231)$, petiole length $(0.1333)$, petiole breadth (0.1431), leaf number (0.1094), leaf area index (0.1496), inflorescence length (0.1118) and cormel length (0.1303). Cormel length showed highly positive and direct effect (1.2414) on yield per plant in genotypic level. It also showed positive and indirect effect via plant height (0.3861), petiole length $(0.1045)$, petiole breadth (0.2656), leaf length (0.1284), leaf number (0.1974), inflorescence length (0.2071), corm length (0.2643), corm breadth (0.3119), cormel number (0.1392). In genotypic level cormel breadth exhibited very low and positive direct effect (0.0940) on yield per plant. It showed also indirect and positive effects via plant height (0.4621), petiole length $(0.1222)$, petiole breadth (0.3135), leaf length $(0.1547)$, leaf number (0.2384), leaf area index (0.1295), inflorescence length (0.2111), corm length (0.1028), corm breadth (0.1183), cormel number (0.1020) and cormel length (0.3414). 


\subsection{At phenotypic level}

At phenotypic level plant height exhibited positive direct effect on yield per plant (0.2823). Low and positive indirect effects also found through petiole length $(0.1252)$, petiole breadth $(0.1924)$, leaf breadth (0.2328), leaf number (0.4058), inflorescence length (0.2009), peduncle length (0.1040), corm length (0.1221), cormel weight (0.1520) and cormel breadth (0.4102). This character exhibited low and positive direct effect on yield per plant $(0.2322)$ at the phenotypic level. Indirect and positive effects was found via plant height (0.1522), petiole breadth (0.2033), leaf breadth (0.2481), leaf number (0.4375), inflorescence length (0.3030), corm length (0.1351), cormel weight (0.3937) and cormel breadth (0.4611). Petiole breadth showed low and positive direct effect $(0.3400)$ at phenotypic level on yield per plant. Indirect but positive effects also found between plant height (0.1598), petiole length (0.1389), leaf number (0.4064), inflorescence length (0.2621), corm length (0.1388), corm breadth (0.1028), cormel weight (0.1809), cormel breadth $(0.4705)$ and yield. At the phenotypic level leaf length showed very low and positive direct effect $(0.0362)$ on yield per plant. Indirect but positive effects was also found between the characters of plant height (0.1971), petiole length $(0.1728)$, petiole breadth $(0.2794)$, leaf breadth (0.3215), leaf number (0.1726), inflorescence length (0.3472), corm length (0.1812), corm breadth (0.1333), cormel number (0.2510), cormel weight $(0.2273)$, cormel length $(0.1114)$ and cormel breadth (0.6153) with yield. Leaf breadth showed low and positive direct effect (0.0176) on yield per plant in genotypic level. Indirect and positive effects were observed through plant height $(0.2268)$, petiole breadth (0.1674), leaf number (0.1669), peduncle length (0.1282), corm length (0.1072), corm breadth (0.1928), cormel number (0.1494), cormel weight (0.1277) and cormel length (0.4370).

Leaf breadth showed positive and direct effects $(0.4034)$ at the phenotypic level on yield per plant. It showed indirect but positive effects via plant height $(0.1629)$, petiole length $(0.1428)$, petiole breadth (0.2142), leaf length $(0.0289)$, leaf number (0.4255), inflorescence length (0.2880), corm length (0.1394), corm breadth (0.1021), cormel weight $(0.4150)$ and cormel breadth (0.4815). Leaf number showed positive direct effect $(0.4014)$ on yield per plant in a phenotypic level. It also showed positive and indirect effects via plant height (0.2854), petiole length (0.2531), petiole breadth (0.3835), leaf breadth $(0.4276)$, inflorescence length (0.1668), corm breadth (0.2206), cormel number (0.1768), cormel weight (0.3770), cormel length (0.1801) and cormel breadth (0.9762). Leaf area index exhibited very low positive direct effect $(0.0226)$ on yield per plant in phenotypic level. It showed positive indirect effect between plant height (0.3388), petiole length $(0.2415)$, petiole breadth $(0.3671)$, leaf breadth (0.2300), leaf number (0.1954), inflorescence length (0.4017), corm length (0.1080), corm breadth (0.2158), cormel number (0.1904), cormel weight (0.7829) and cormel breadth (0.8220). Inflorescence length showed low and positive direct effect (0.3404) on yield per plant in phenotypic level. It also showed positive indirect effects via plant height (0.1666), petiole length (0.2067), petiole breadth (0.2618), leaf breadth (0.3413), leaf number (0.1967), corm breadth (0.3769), cormel number (0.2716), cormel weight (0.5017), cormel length (0.1294) and cormel breadth (0.8533). In phenotypic level peduncle length exhibited very low and positive direct effect on yield per plant (0.1397). Peduncle length also showed positive and indirect effects between plant height $(0.3024)$, petiole breadth (0.1836), leaf breadth $(0.2743)$, leaf number (0.3532), inflorescence length (0.1906), corm length $(0.1060)$, corm breadth $(0.3539)$, cormel number (0.2573), cormel weight (0.7829), cormel length $(0.1277)$, cormel breadth $(0.5715)$ and yield per plant. In phenotypic level corm length exhibited low and positive direct effect on yield per plant (0.1963). It showed positive and indirect effects via plant height $(0.1756)$, petiole length (0.1598), petiole breadth $(0.2403)$, leaf breadth (0.2864), leaf number (0.1846), corm breadth (0.1960), cormel number (0.1567), cormel weight (0.3512), cormel length (0.1417) and cormel breadth (0.7852). It also showed positive and indirect effects yield per plant via plant height 
(0.2770), petiole breadth (0.1819), leaf number (0.1335), inflorescence length (0.1398) and cormel length (0.5040).

In phenotypic level corm breadth showed positive and direct effect on yield per plant (0.3426). It also showed indirect and positive effects via petiole breadth $(0.1020)$, leaf breadth $(0.1202)$, leaf number (0.2585), inflorescence length (0.3745), peduncle length (0.1003), corm length (0.1123), cormel weight (0.2432) and cormel breadth (0.3883). In phenotypic level corm weight exhibited very low and positive direct effect (0.0430) on yield per plant. It showed positive and indirect effects through plant height (0.1920), petiole length $(0.1725)$, petiole breadth $(0.2641)$, leaf breadth (0.3187), leaf number (0.2328), inflorescence length (0.2077), corm length (0.2450), corm breadth (0.2069), cormel number (0.1640), cormel weight (0.3770), cormel length (0.1497) and cormel breadth (0.8376). Cormel weight showed very high and positive direct effect on yield per plant (0.7601) in phenotypic level. It also exhibited positive and indirect effects via petiole length (0.1203), leaf breadth $(0.2203)$, leaf number (0.1991), inflorescence length (0.2247), peduncle length $(0.1039)$, corm breadth $(0.1117)$ and cormel breadth (0.3045). Phenotypically, cormel length exhibited low and positive direct effect (0.1749) on yield per plant. It also showed positive indirect effect through plant height (0.1194), petiole length $(0.1119)$, petiole breadth (0.1635), leaf breadth $(0.2005)$, leaf number (0.4094), inflorescence length (0.2519), corm length (0.2775), corm breadth $(0.1127)$, cormel number (0.1097), cormel weight (0.2303) and cormel breadth (0.5730). In phenotypic level cormel breadth showed highly strong positive direct effect $(0.7828)$ on yield per plant. It also showed positive and indirect effects via plant height (0.1479), petiole length (0.1368), petiole breadth $(0.2043)$, leaf breadth $(0.2481)$, leaf number (0.5006), inflorescence length (0.3813), corm length (0.1969), corm breadth (0.1699), cormel number $(0.1333)$, cormel weight $(0.2957)$ and cormel length (0.1280).

In any breeding programme, improvement of the nature and pattern of crop plants depend upon the nature and magnitude of variation and extent to which the desirable characters are heritable (Dudley and Moll, 1969). For genetic improvement of any crops breeders requires information on nature and magnitude of variation in the existing population the high potentiality of the genetic variability as experienced by a character is the main concern of breeders and their magnitude can be measured from the study of genetic coefficient of variability. According to Offori and Bernett-larteg (1995), morphological characters are important diagnostic features for distinguishing among genotypes. They may serve as genetic markers which facilitate and speed up selection in crop improvement programmes

In genotypic level, highest direct effect was observed by cormel length followed by plant height. Inflorescence had the highest indirect effect via cormel length and plant height toward yield per plant followed by leaf area index via plant height. The residual effect was 0.3043 which indicates that nearly $69.5 \%$ yield was contributed by the studied characters. Plant height, petiole breadth, corm breadth, cormel number and cormel length had the good effect on yield per plant. In the phenotypic level, highest direct effect was found for cormel breadth followed by cormel weight toward yield per plant. Highest indirect effect was expressed by leaf number via cormel breadth followed by LAI and corm weight. Thus cormel weight and cormel breadth influenced yield per plant directly in greater magnitude. Residual effect was 0.4874 means about $51.26 \%$ contributed yield by this characters. The information of the path analysis of edible aroids is very rare. However, Mahonkumar et al. (1990) reported that weight of cormels was the strongest force determining yield in taro. Goenaga (1994) reported that weight of mother cormels and weight of cormels had highest direct and indirect effects and was the strongest forces influencing yield at both phenotypic and genotypic levels and this could be used as selection criteria in breeding programs through path analysis in tanier crops. Pandey et al. (1996) reported that 
weight of mother cormels and weight of cormels had the highest direct and indirect effects and were the strongest forces influencing yield at both phenotypic and genotypic levels and thus could be used as selection criteria in breeding program. Dwivedi and Sen (1999) studied path coefficient analysis on thirty genotypes of taro and revealed that corm weight had the highest direct effect on the cormel yield and positive indirect effect via girth of main sucker. Highest negative direct effect was recorded by length of main sucker. Selection based on corm weight and higher girth of main sucker will be efficient to maximize the cormel yield in taro. Tsegaye et al. (2006) studied thirty sweet potato genotypes for path coefficient analysis. They also revealed that individual storage root weight, number of storage roots per plant and harvest index were the most important determinants of storage root yield. It could be concluded that due to the high estimated positive correlation and positive direct effect of individual storage root weight and harvest index on storage root yield, these trait would be most suitable for indirect selection in sweet potato improvement programs that aim at increasing storage root yield. Cheema et al. (2007) studied path analysis and revealed that number of cormels per plant, corm weight and length had direct and positive effects while number of corms per plant had indirect and positive effects on total yield per plant.

\section{Conclusion}

It can be concluded that the characters plant height, petiole breadth, corm breadth, cormel number and cormel length exhibited direct effect on yield per plant at the genotypic level and holds the highest merit to be selected in the breeding programme towards improving the taro plants. Residual efects showed that characters contribute their effect $52 \%$ phenotypically and $70 \%$ genotypically to yield.

\section{Acknowledgements}

Thanks to the University Grant commission, Dhaka-1207, Bangladesh for nominating me as $\mathrm{Ph}$. D fellow through financial support. Also acknowledge the Ministry of Science and Information \& Communication Technology, Bangladesh for partly financial support to conduct the study.

\section{References}

Cheema, D. S., Singh, H., Dhatt, A. S. and Gang, N. 2007. Studies on gentic variability and correlation for yield and quality traits in Arvi (Colocasia esculenta Schott). International Society For Horticultural Science, Acta Horticulturae, 752.

Cochran, W. G. and Cox, G. M. 1960. Experimental design. $2^{\text {nd }}$ edition. John Willey and Sons, Inc. New York.

Coursey, D. G. 1968. The edible aroids. World Crops, 20(4): 25-30.

Dudly, J. W. and Moll, R, H, 1969. Introduction and use of estimates of heritability in Soyabean. Agronomy Journal, 47: 314 318.

Dwivedi, A. K. and Sen, H. 1999. Correlation and path coefficient studies in taro (colocasia esculenta var. antiquorum). Journal of Root Crops, 25(1): 51 - 54.

Fisher, R, A., Immer, R. R. and Tedin, O. 1932. The genetic interpretation of statistics on the third degree in the inheritance of quantitative inheritance. Genetics, 17: 107 - 124.

Goenaga, R, 1994. Growth, nutrient uptake and yield of tanier (Xanthosoma spp.) grown under semiarid conditions. Journal of Agriculture of the University of Puerto Rico, 78(3 - 4): 87 - 98.

IPGRI, 1999. Descriptors for taro (Colocasia esculenta). IPGRI, Rome, Italy. 26 - 42 pp.

Mather, K. 1949. Biometrical genetics, Dover publication. Inc. New York.

Mohonkumar, C. R., Sarawathy, $\mathrm{P}$ and Sadanandan, N. 1990. Correlation and path coefficient analysis on yield and yield component in Taro. Journal of Root Crops, 16(2): 140 -141. 
Montgomery, E. G. 1911.Correlation studies in corn. Nebraska Agricultural Experimental Station, Annual Report, 24: 108 - 159.

Offori, I. and Bernett-Larteg. 1995. Variation in morphological characteristics in a collection of cowpea (Vigna unguiculata L.Walp) land races. Legon Agric Research and Extension Journal, 4: 77 - 85.

Pandey, G., Dhobal, V. K. and Sapra, R. L. 1996. Genetic variability, correlation and path analysis in Taro (Colocasia esculenta). Journal of Hill Research (India), 9(2): 299 - 302.

Panse, V. G. and Sukhatme, P. V. 1978. Statistical Methods for Agricultural workers. Indian Council of Agricultural workers. Indian council of Agricultural Research, New Delhi, India.

Singh, R. K. and Chaudhary, B. D. 1977. Biometrical methods in quantitative genetic analysis .Kalnani publishers, New Delhi, India. 49 -100 pp.

Tsegage Engida, Nigussie, E. V. Dechassa and Devakara, Sastry, 2006. Correlation and path analysis in sweet potato and their implication s for clonal selection. Journal of Agronomy, 5 (3): 391 - 395.

Wright, S. 1921. Correlation and causation. Journal of Agriculture Research, 20: 557 585.

Wright, S. 1923. The theory of path coefficient a reply to Niles criticism. Genetics, 8: 23. 
